\title{
Comparison of diagnostic clinical samples and environmental sampling for enterovirus and parechovirus surveillance in Scotland, 2010 to 2012
}

\author{
H Harvala (heli.simmonds@hotmail.com) ${ }^{1,2}$, J Calvert ${ }^{1}$, D Van Nguyen ${ }^{1}$, L Clasper ${ }^{1}$, N Gadsby², P Molyneaux ${ }^{3}$, K Templeton Tem, $^{1,2}$ \\ C McWilliams Leitch ${ }^{1}$, P Simmonds ${ }^{1,2}$ \\ 1. Infection and Immunity, Roslin Institute, University of Edinburgh, Edinburgh United Kingdom \\ 2. Specialist Virology Centre, Royal Infirmary Edinburgh, Edinburgh United Kingdom \\ 3. Department of Medical Microbiology, Aberdeen Royal Infirmary, Aberdeen United Kingdom
}

Citation style for this article:

Harvala H, Calvert J, Van Nguyen D, Clasper L, Gadsby N, Molyneaux P, Templeton K, McWilliams Leitch C, Simmonds P. Comparison of diagnostic clinical samples and environmental sampling for enterovirus and parechovirus surveillance in Scotland, 2010 to 2012. Euro Surveill. 2014;19(15):pii=20772. Available online: http:// www.eurosurveillance.org/ViewArticle.aspx?Articleld $=20772$

Article submitted on 04 March 2013 / published on 17 April 2014

Human enteroviruses (EV) and parechoviruses (HPeV) within the family Picornaviridae are the most common causes of viral central nervous system (CNS)associated infections including meningitis and neonatal sepsis-like disease. The frequencies of EV and HPeV types identified in clinical specimens collected in Scotland over an eight-year period were compared to those identified in sewage surveillance established in Edinburgh. Of the 35 different EV types belonging to four EV species (A to D) and the four HPeV types detected in this study, $\mathrm{HPeV}_{3}$ was identified as the most prevalent picornavirus in cerebrospinal fluid samples, followed by species B EV. Interestingly, over half of EV and all HPeV CNS-associated infections were observed in young infants (younger than three months). Detection of species A EV including coxsackievirus $A 6$ and EV71 in clinical samples and sewage indicates that these viruses are already widely circulating in Scotland. Furthermore, species C EV were frequently identified EV in sewage screening but they were not present in any of 606 EV-positive clinical samples studied, indicating their likely lower pathogenicity. Picornavirus surveillance is important not only for monitoring the changing epidemiology of these infections but also for the rapid identification of spread of emerging EV and/or HPeV types.

\section{Introduction}

While poliovirus (PV) eradication is approaching its goal, other picornaviruses, including enteroviruses (EV) and parechoviruses (HPeV) are growing in clinical importance. In Europe, EV are the most common cause of viral meningitis in children and young adults, and the newly emerging HPeV type 3 is proving to be an important cause of central nervous system (CNS) infection in neonates [1]. At the same time, the incidence of hand, foot and mouth disease (HFMD) and severe $\mathrm{EV}_{71}$ infections in children is increasing in Asia, and the virus strain(s) responsible could potentially be imported to Europe [2].

Enteroviruses were traditionally divided into polioviruses (PV, three serotypes), coxsackie $A$ viruses (CAV, 23 serotypes), coxsackie B viruses (CBV, six serotypes) and echoviruses (E, 28 serotypes) based on their antigenic and pathogenic properties in humans and laboratory animals [3]. However, this biological division has been replaced by a molecular classification based on VP1 sequencing, and more recently discovered EV types have been numbered in the order of their identification (50 numbered EV types by 1 April 2014) [4]. Sequence analysis of enteroviruses furthermore showed evidence for a deeper grouping into four species A-D (EV-A to D) that cuts across these previous biologically defined categories. EV within species $B$ (all echoviruses, $C_{B V}-6$ and $\left(A V_{9}\right.$ ) are the most commonly identified cause of viral meningitis in Europe, whereas viruses causing HFMD generally fall within species $A$.

The first two serologically distinct HPeV types, originally described as echoviruses (E22 and E23) in the Enterovirus genus, were discovered over 50 years ago (reviewed in [5]). However, they were renamed as HPeV and reclassified into their own Parechovirus genus in 1999 based on their molecular and biological properties [6]. Since then, a further $14 \mathrm{HPeV}$ types $\left(\mathrm{HPeV}_{3}\right.$ to 16) have been discovered, with HPeV type 3 specifically associated with severe neonatal CNS-infections [5].

Laboratory detection of EV or HPeV generally uses molecular methods such as reverse transcription PCR (RT-PCR), which are faster and more sensitive than viral cell culture. The 5'non-coding region (NCR) is the most conserved region among $\mathrm{EV}$ and $\mathrm{HPeV}$, and is therefore targeted in many diagnostic screening procedures. Unfortunately, sequences from this region provide little or no information on the (sero)type of the infecting 
virus, and sequencing of a structural gene region such as $\mathrm{VP}_{1}$ is required to enable type identification for EV and $\mathrm{HPeV}[5,7]$. Molecular typing is important for ensuring that PVs are not re-introduced into the countries where they have already been eradicated and for more general surveillance and epidemiological purposes. However, as a surveillance method, such screening has severe limitations given that only a very small subset of EV infections are diagnosed through referral of clinical samples for virological testing. In the United Kingdom (UK), EV detection is clinically focussed on neurological disease including viral meningitis and done very rarely in cases of, for example, HFMD since these patients are not normally hospitalised. Analysis of sewage for the presence of EV and HPeV provides an alternative and additional surveillance method without referral bias, and complements the clinical data with a potentially more accurate representation of virus types circulating in the community.

In the current study we have performed comprehensive typing of EV and HPeV detected in diagnostic clinical samples from the east of Scotland over a three-year period. Frequencies of EV and HPeV types identified in 2010 to 2012 were compared with those identified in previous years (2005 to 2010 ; [1]) to provide a longer term indication of their incidences and age distributions. They were also compared with types identified as circulating in the community through sewage surveillance in Edinburgh and surrounding areas to provide a more complete description of the clinical epidemiology of $\mathrm{EV}$ and $\mathrm{HPeV}$ infections.

\section{Methods}

\section{Cerebrospinal fluid samples}

A total of 3,415 cerebrospinal fluid (CSF) samples referred to the Specialist Virology Centre in Edinburgh for virology testing during the three-year study period from 2010 to 2012 were included in this study; these were compared to previous results from 3,957 CSF samples obtained between 2005 and 2009 [1]. EV screening was done by separate $R T$ and $P C R$ reactions [8] until real-time EV RT-PCR (modified from [9]) was introduced into routine use in the beginning of 2009 and combined further with real-time human parechovirus (HPeV) RT-PCR in 2011 [10]. CSF samples collected before 2011 were tested retrospectively for HPeV by RT-PCR $[1,11]$. In addition, 82 EV-positive and $10 \mathrm{HPeV}$ positive CSF samples were referred for typing from elsewhere in Scotland.

\section{Other clinical samples}

Eleven of 19 EV-positive vesicular swab samples obtained from individuals with HFMD (11 of them were hospitalised) in Edinburgh between 2010 and 2011 as well as 185 EV-positive clinical specimens (64 faecal samples, 12 vesicle swabs and 109 respiratory samples) and seven HPeV-positive samples (four faecal and three respiratory samples) submitted for typing from elsewhere in Scotland between 2010 and 2012 have been included in this study. Samples submitted for typing from elsewhere in Scotland were screened using EV primers, which are also known to detect human rhinovirus (HRV).

\section{Clinical samples positive for enterovirus and parechovirus}

Samples were anonymised and archived according to the protocol approved by the Lothian Regional Ethics Committee (08-S11/02/2). Extracted RNA was amplified by a combined RT- and first-round PCR using the High Fidelity Superscript III Platinum Taq (Invitrogen, UK) followed by a second amplification reaction with nested primers specific for species $B V_{1}$ sequences [12]. If negative, the PCR was repeated with species $A$ $\mathrm{VP}_{1}$ primers and with general VP4 primers; these also amplify HRV sequences and are also used for HRV typing. Positive HPeV samples were amplified in the $\mathrm{VP}_{3} /$ VP1 region [11].

\section{Sewage specimens}

A total of 40 waste water samples were collected approximately a week apart in twelve consecutive months (June 2009-May 2010) from the Veolia Wastewater Treatment works, which processes all waste water from the sewage system and road run-off in the urban and surrounding areas of Edinburgh (population size approximately 650,000 people). A total of 100 $\mathrm{g}$ of solid waste ( $50 \mathrm{~g} \pm 2.5 \mathrm{~g}$ from two sampling sites) was resuspended in $200 \mathrm{~mL}$ sterile phosphate buffered saline by vortexing for at least one minute to remove any particulate matter from the sample. Centrifugation and size fractionation using filters (Millipore, Pall, UK) of different pore sizes were used to enrich samples as previously described [13]. RNA was extracted from the filtrate using the Qiagen extraction kit (Qiagen, UK) and then reverse-transcribed using the Reverse Transcription System (Promega, UK). Amplification of CDNA was performed by nested PCRs using different primers for each EV species and HPeV [11,12].

\section{Sequencing}

Amplified $\mathrm{VP}_{3} / \mathrm{VP}_{1}$ and $\mathrm{VP}_{4}$ regions from clinical and sewage specimens were directly sequenced using the BigDye Terminator kit (Applied Biosystems, Warrington, UK) using inner primers $[11,12]$. Sequences were aligned using SSE version 1.1 (http://www.virusevolution.org/Downloads/Software/).

\section{Results}

Enterovirus and parechovirus infections

Screening of the 3,415 archived CSF samples identified EV RNA in a total of 150 individual specimens obtained from 150 different individuals and HPeV RNA in a total of 35 specimens obtained from 35 different individuals. EV were detected throughout the three-year study period: 41 of $1,043(4 \%)$ in 2010,69 of $1,172(6 \%)$ in 2011 and 40 of 1,200 (3\%) in 2012 (Figure 1A). Marked annual changes in the incidence of HPeV infections were in keeping with our previous five-year report [5]; 


\section{FIGURE 1}

Detection frequency of enterovirus and human parechovirus in cerebrospinal fluid samples, by time (A) and by age (B), Edinburgh, 2005-2012 ( $\mathrm{n}=7,372)$

A.

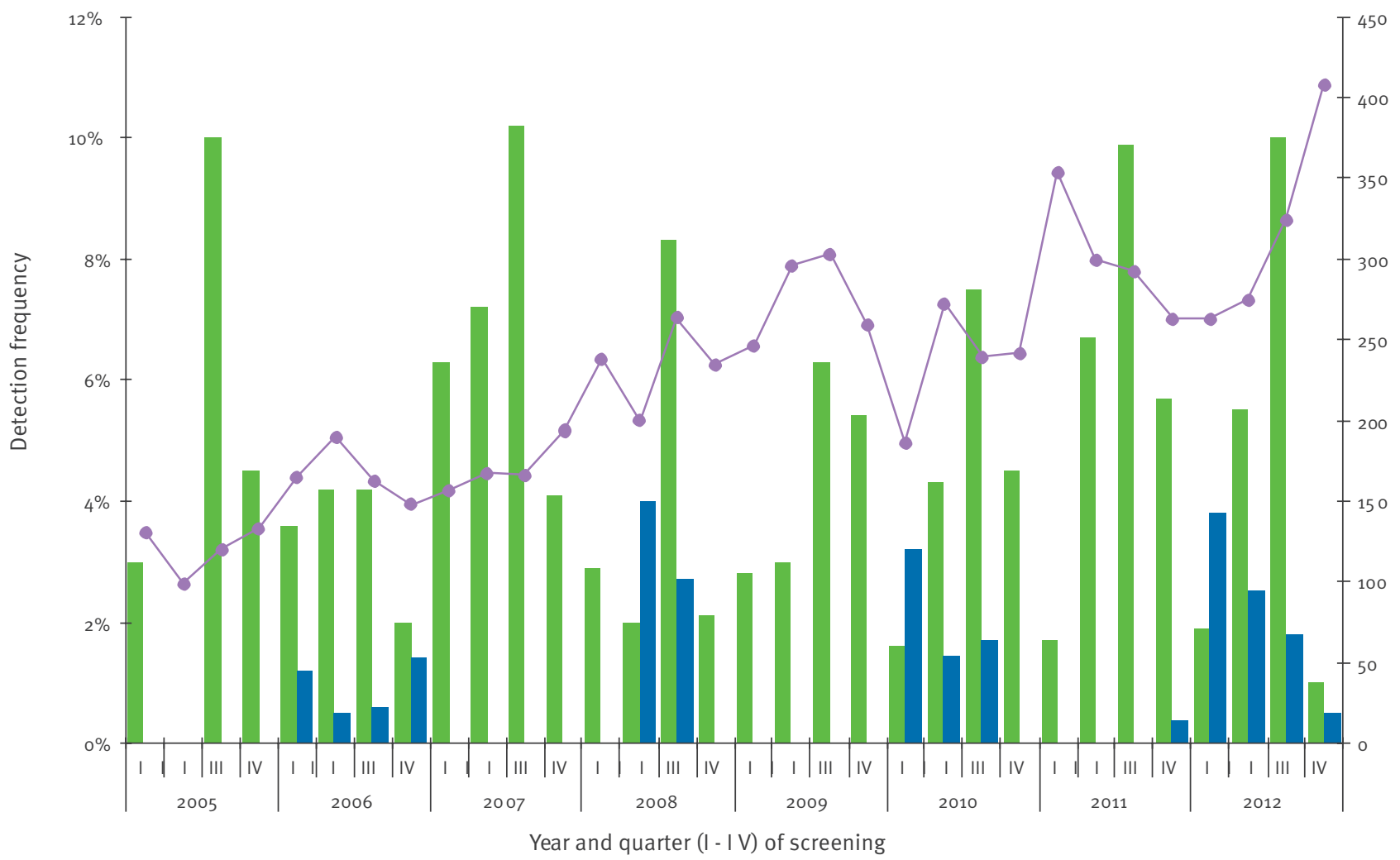

B.
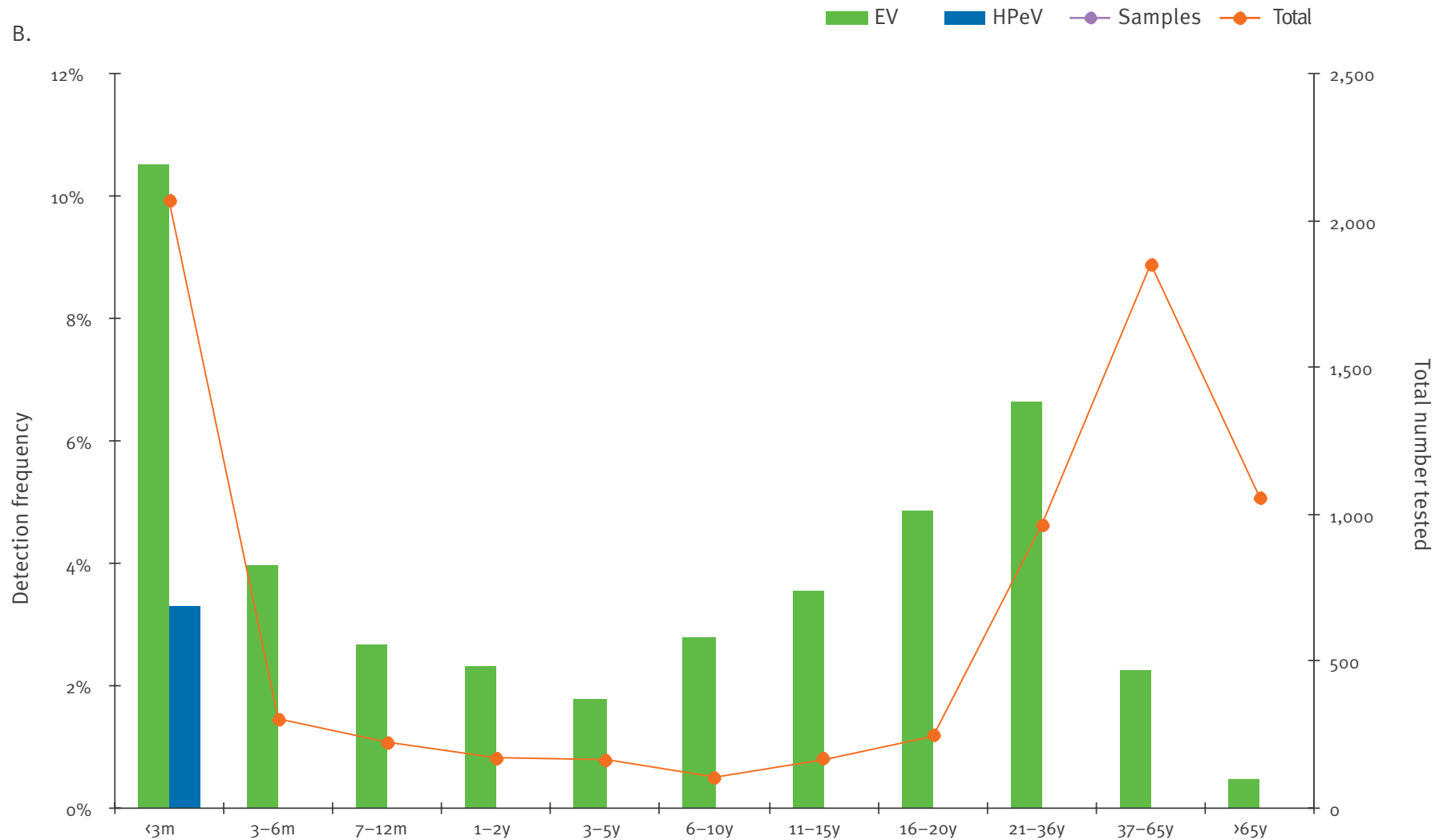

EV: enterovirus; HPeV: human parechovirus; m: months; y: years.

I: January, February, March; II: April, May, June; III: July, August, September; IV: October, November, December. 
Distribution of enterovirus and human parechovirus types in cerebrospinal fluid samples, Scotland, 2005-2012 (n=404)

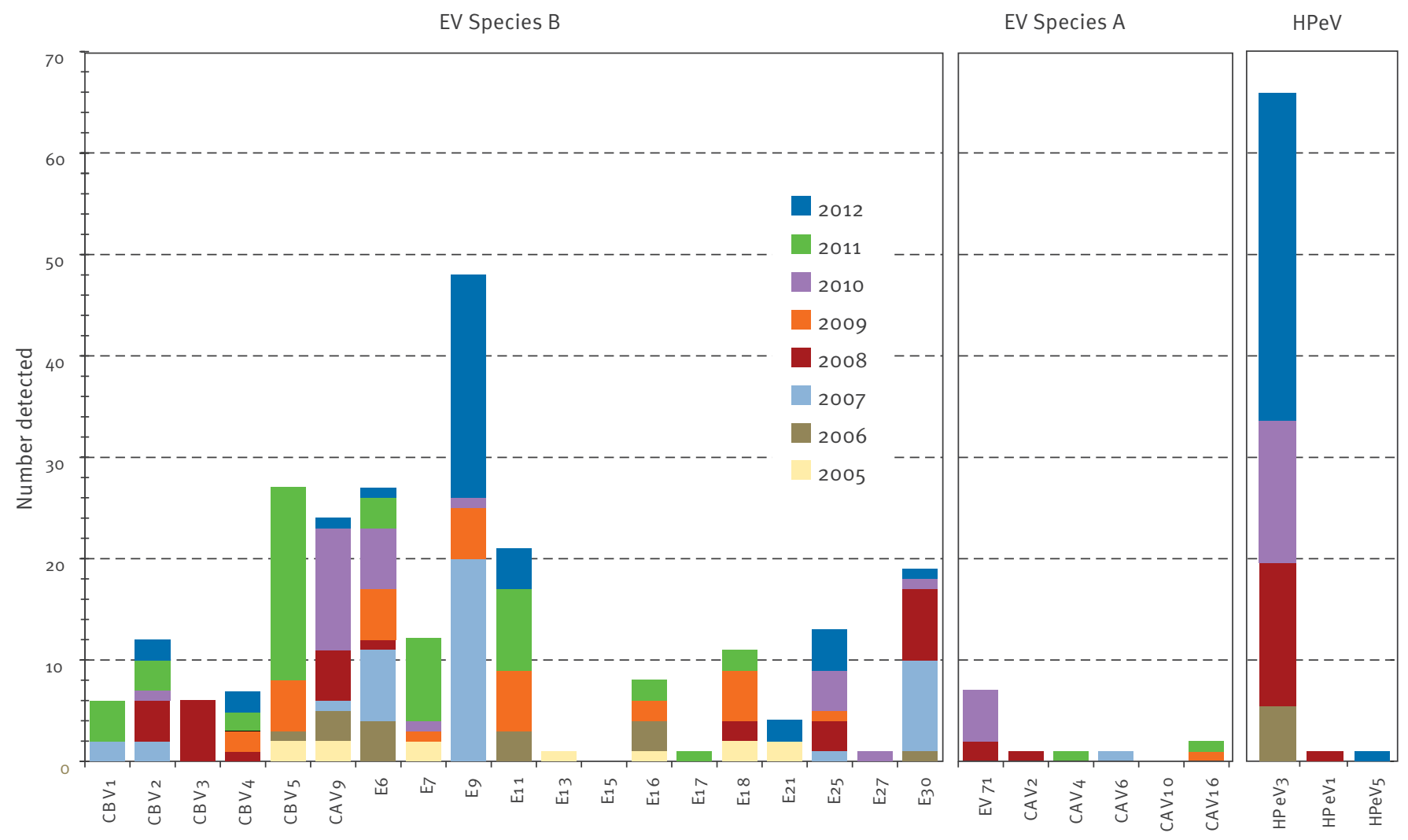

CAV: coxsackie A virus; EV: enterovirus; HPeV: human parechovirus.

most HPeV infections were recorded in even years, 14 in $2010(1.3 \%)$ and 23 in $2012(1.8 \%)$, whereas screening of the total 3,513 CSF samples collected during the odd years (2,342 in 2005, 2007 and 2009 as previously reported [1] and 1,171 in 2011 [this study]) resulted in only a single $\mathrm{HPeV}$ detection (Figure $1 \mathrm{~A}$ ). The highest frequency of EV infections was seen in young children under the age of three months $(217 / 2,066,11 \%)$, whereas individuals infected with $\mathrm{HPeV}$ were exclusively infants under the age of three months $(58 / 2,066$, $3 \%$; Figure $1 \mathrm{~B}$ ).

\section{Enterovirus and parechovirus type identification}

All 606 EV-positive clinical samples and $75 \mathrm{HPeV}$ positive samples were subjected to genotyping, including the sequences obtained and typed in 2005 to 2009 in our previous study [1]. As a result, a high proportion of these (uncultured) clinical samples could be directly typed for EV (498/606; $82 \%)$, specifically $82 \%$ of CSF samples (336/410), 78\% of faecal samples (50/64), $83 \%$ of vesicle samples $(19 / 23)$ and $85 \%$ of respiratory samples (93/109). EV-positivity of untypeable samples was confirmed by 5'UTR PCR. For HPeV, all 75 5'UTR screening-positive samples could be amplified and typed in the $V P_{3} / V P_{1}$ region.
Cerebrospinal fluid samples

A total of 19 different species B and six species A EV serotypes were detected in CSF samples in this study. The six most frequently detected EV types were E9 (57/336; 17\%), CAV9 (35/336; 10\%), CBV 5 (35/336; $10 \%)$, E6 (29/336; 9\%), E11 (23/336; 7\%) and $\mathrm{E}_{30}$ (20/336; 6\%) corresponding to $59 \%$ of CNS-associated EV infections, along with occasional detections of species A serotypes CAV2, CAV4, CAV6, CAV10, CAV16 and

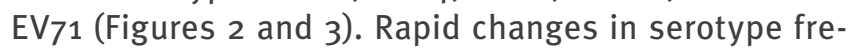
quencies were observed. Almost all EV were typed as E9 in 2007, CAV9 in 2010, CBV 5 in 2011 and E9 in 2012. No virus predominated in 2005, 2006, 2008 and 2009. All except two of the HPeV strains identified in CSF samples were $\mathrm{HPeV}_{3}(66 / 68)$, whereas the remaining ones were $\mathrm{HPeV}_{1}$ and $\mathrm{HPeV}_{5}$. Over the eight-year study period, $\mathrm{HPeV}_{3}$ remained the most prevalent picornavirus in CNS-related infections.

\section{Other clinical samples}

A total of 13 different species $B$ and six different species $A$ EV were identified in the 50 successfully typed faecal samples, with $\mathrm{CBV}_{5}$ and $\mathrm{EV}_{71}$ being the most common types (Figure 3). In addition, a high proportion of EV-positive faecal samples (8/50) were identified as HRV as reported previously [14]. It is known that 


\section{FIGURE 3}

Comparison of enterovirus types identified in clinical and sewage samples obtained in Scotland, 2010-2012

Species B

Species A

Species C

D HRV

A. CSF

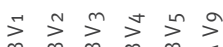

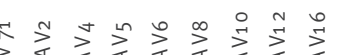

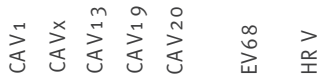

A. CSF

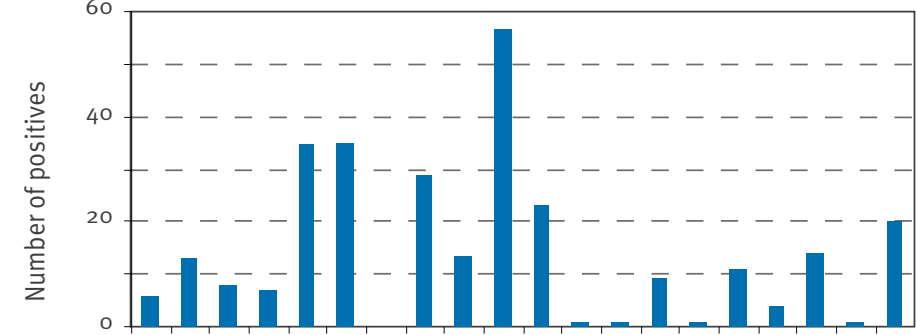

B. Faeces
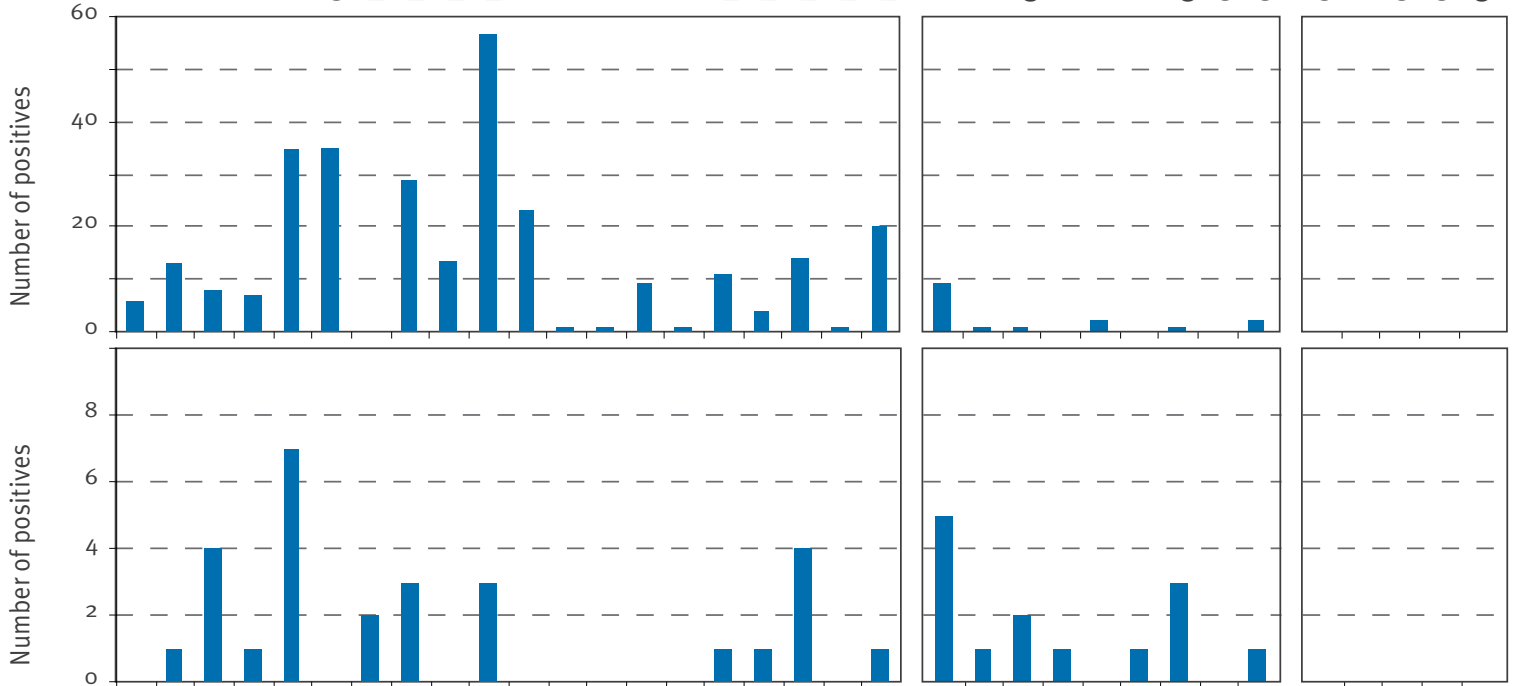

C. Resp.
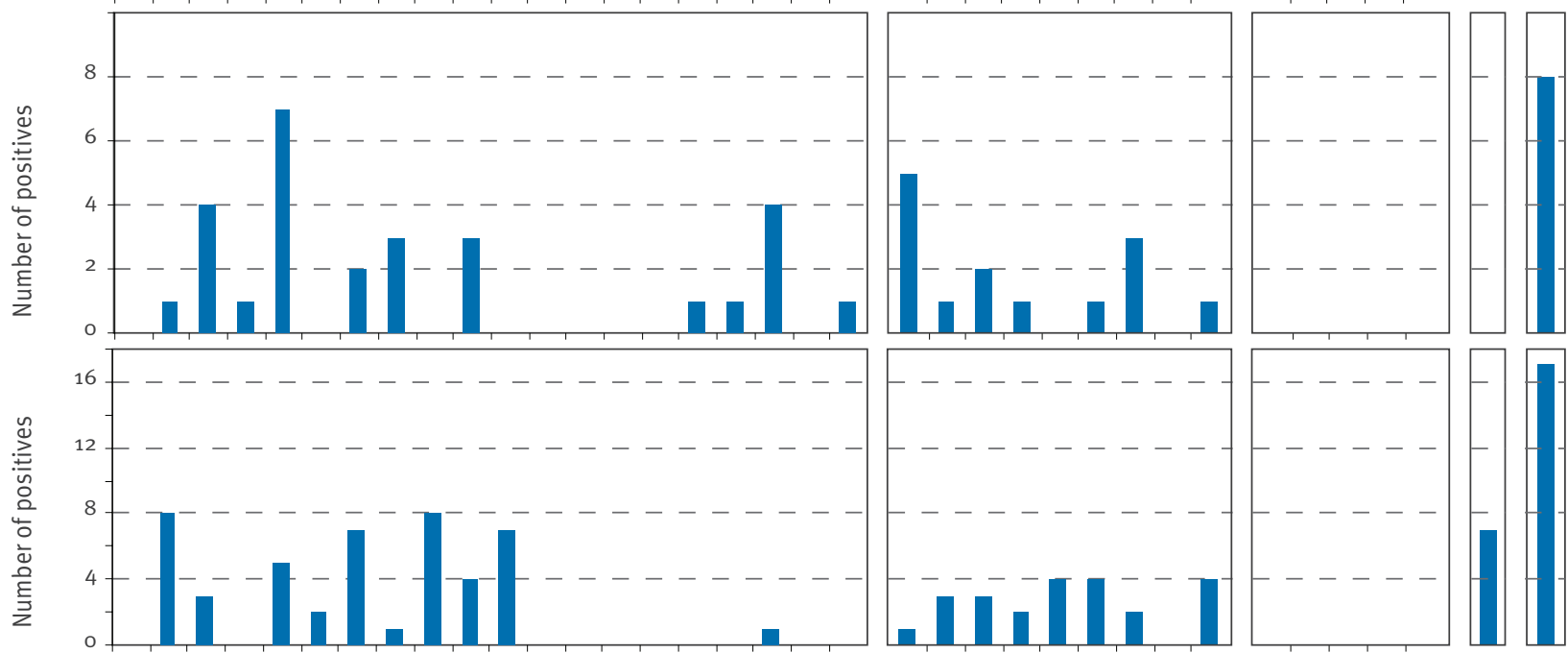

D. Vesicle
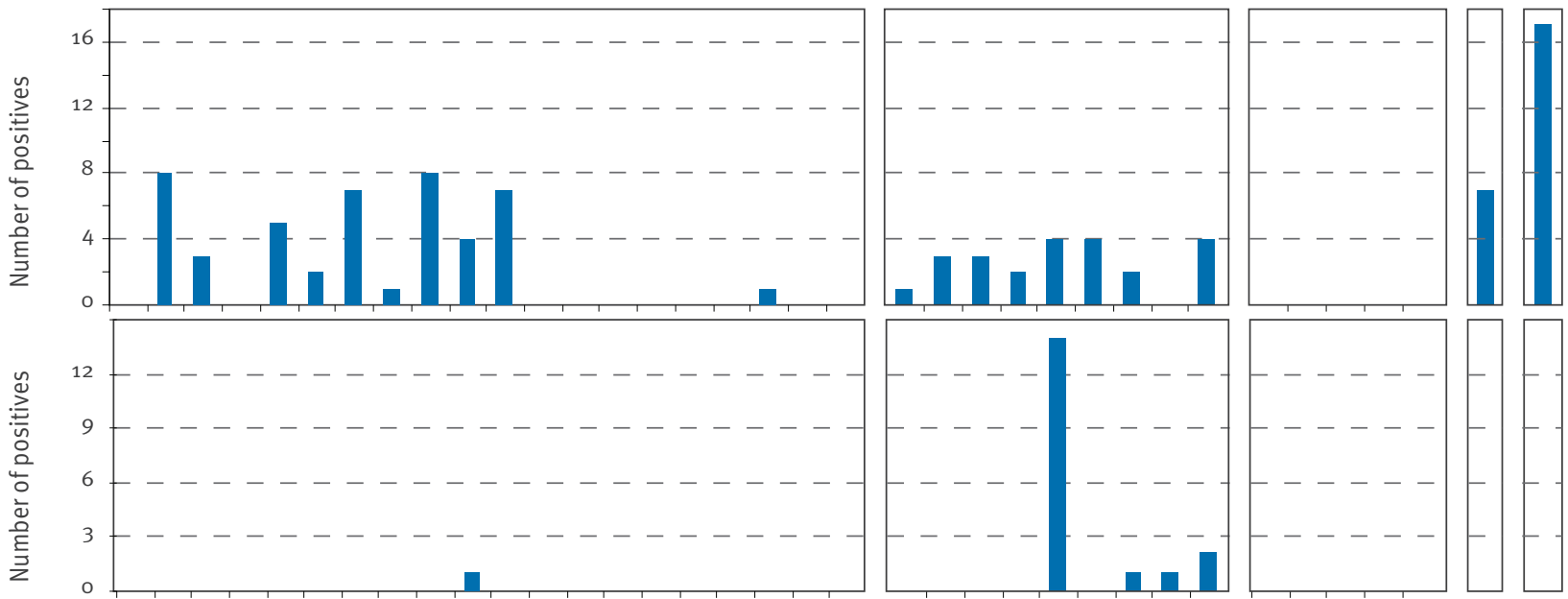

E. Sewage
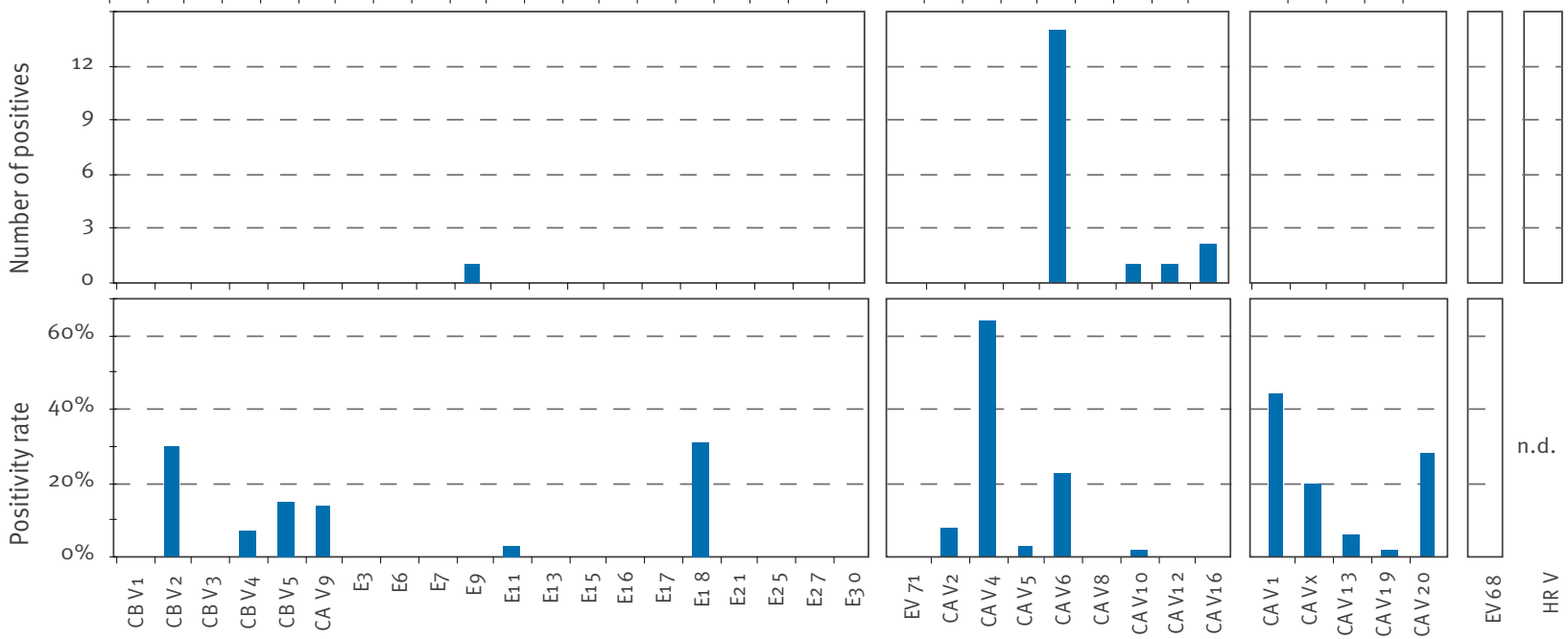

CAV: coxsackie A virus; CSF: cerebrospinal fluid; EV: enterovirus; ND: not determined; HPeV: human parechovirus; HRV: human rhinovirus.

EV primers will detect some HRV [14]. The species A serotype, CAV6 was the most frequently identified variant among the vesicular swabs (14/19). Furthermore, a total of eight individual species A and 10 species B EV types were found in the 93 typed throat swabs, along with EV68 (species D) and several HRV strains. All four HPeV-positive faecal samples were typed as $\mathrm{HPeV}_{3}$, whereas the two HPeV-positive respiratory samples were identified as $\mathrm{HPeV}_{5}$ and one as $\mathrm{HPeV}_{3}$.
Sewage specimens

During a one-year period from June 2009 to May 2010, EV was identified in 37 of 40 sewage samples, and HPeV in 31 of 40 . Samples were screened for EV using four species-specific VP1 primer sets, allowing EV from all species to be identified. However, species D EV were not detected in this study. From 353 cloned sequences obtained, a total of 95 amplicons were identified as species $C$ and by phylogenetic analysis could be identified as CAV1 (42/95), CAV22 (27/95), CAV13 


\section{FIGURE 4}

Human parechovirus typing of sewage samples obtained in Edinburgh, June 2009-May 2010.

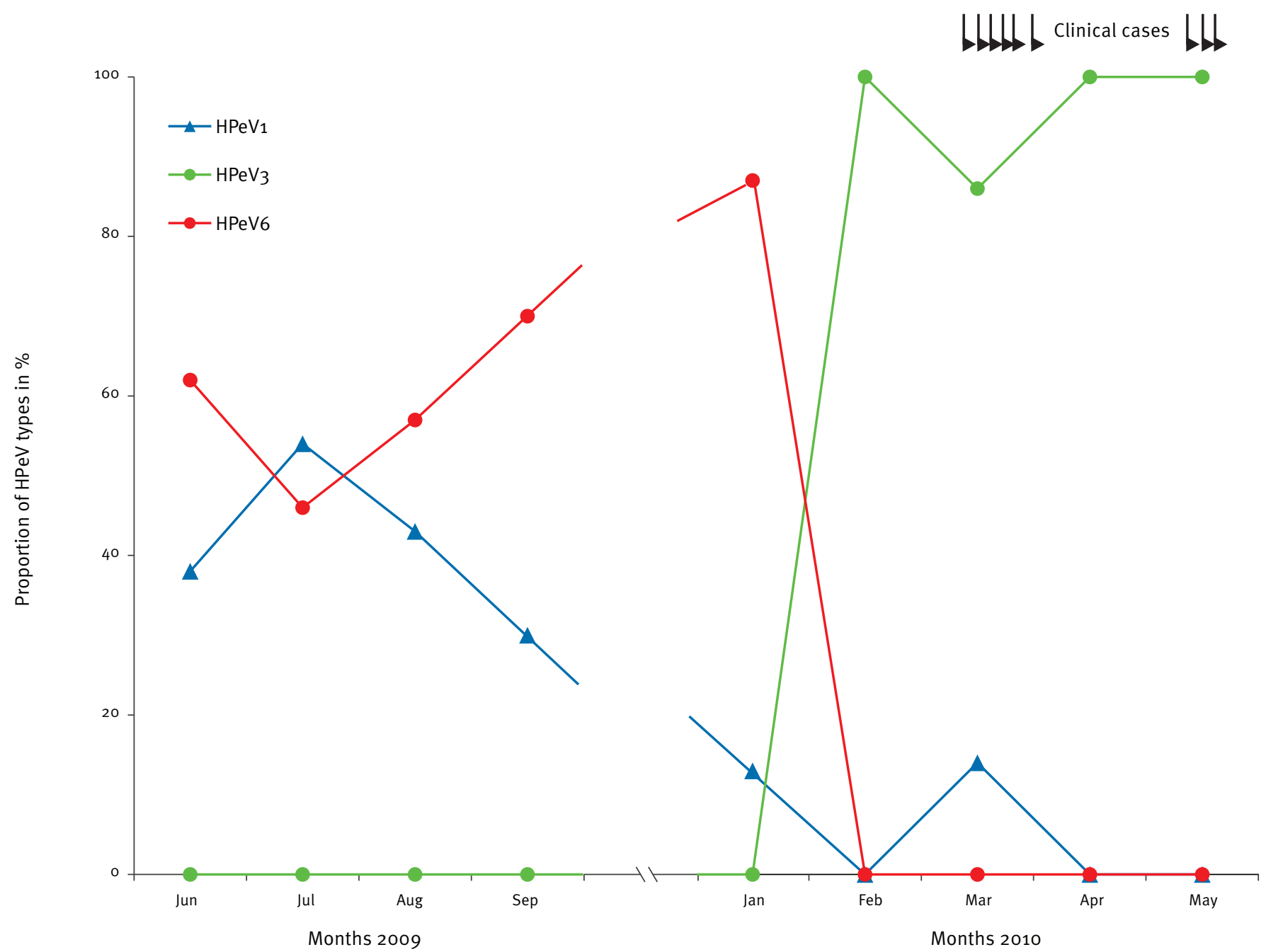

HPeV: human parechovirus.

The appearance of $\mathrm{HPeV}_{3}$ in clinical CSF samples is indicated by the vertical arrows.

(6/95) and CAV19 (2/95) (Figure 3). The remaining 18 sequences clustered together but separately from all known species $C$ types, including the newly identified $\mathrm{EV}_{109}, \mathrm{EV}_{113}$ and $\mathrm{EV}_{116}-118$. Their assignment as a new species $\mathrm{C}$ type will require complete $\mathrm{VP}_{1}$ sequences, which will be the subject of a further study. No PV was detected in sewage.

The 73 species $B$ variants identified from sewage were most closely related to $\mathrm{E}_{18}(23 / 73), \mathrm{CBV}_{2}$ (22/73), $\mathrm{CBV}_{5}$ (11/73), CAV9 (10/73), CBV4 (5/73) and E11 (2/73), whereas 61 species $A$ strains clustered closely with $\mathrm{CAV}_{4}$ (39/61), CAV6 (14/61), CAV2 (5/61), CAV5 (2/61) and $\mathrm{CAV}_{10}(1 / 61)$. Interestingly, $\mathrm{CAV}_{9}$ and $\mathrm{E}_{18} 8$ were also among the most common EV detected in clinical specimens during the same time. On the other hand, although CAV4 was commonly identified in sewage, it was an example of EV-A, which has very rarely been identified in clinical specimens.
The presence of $\mathrm{HPeV}$ was determined using primers which amplify the $\mathrm{VP}_{3} / 1$ region, enabling genotype identification. In total, $124 \mathrm{HPeV}$ sequences were found; $\mathrm{HPeV}_{3}$ being the most common type (62/124), followed by HPeV6 (36/124) and HPeV1 (26/124). $\mathrm{HPeV}_{3}$ appeared in sewage one week before the first clinical case was diagnosed with $\mathrm{HPeV}_{3}$ infection (Figure 4).

\section{Discussion}

This study describes the epidemiology of EV and HPeV infections based on CSF screening of hospitalised individuals over an eight-year period in Edinburgh, combines these data with local sewage surveillance, and samples submitted for typing from elsewhere in eastern Scotland. Because of the similar sensitivity of PCR for all EV species (A to D) [10], our rates of detection are not influenced by variability and insensitivity of viral cell culture, an important drawback of previous EV 
surveillance reports that relied mainly on virus isolation. Similarly, the use of PCR provides a better reflection of the relative importance of $\mathrm{HPeV}$ in CNS-related disease.

\section{Diversity of enteroviruses}

Over the eight-year study period, 326 individuals presented with CNS-associated EV infection with the diagnosis established by virus detection in CSF. Interestingly, more than half of these EV infections $(58 \%)$ were observed in infants under the age of three months, a higher proportion than we found in our surveillance study covering only the first five-year period [1]. This increasing percentage may reflect decreasing seroprevalence against EV infections in Europe [15]. Surveillance data from several countries have shown that approximately $29 \%$ to $44 \%$ of CNS-associated EV infections occur in young children under the age of one year $[16,17]$, but specific data on young infants are rare.

The six most common EV serotypes (all from species B) identified in CSF samples collected in Edinburgh were $\mathrm{E} 9, \mathrm{CAV}_{9}, \mathrm{CBV}_{5}, \mathrm{E} 6, \mathrm{E}_{11}$ and E30. Four of these (E6, E9, E30 and $(A V 9)$ have been among the most abundant serotypes isolated from clinical specimens in the UK and elsewhere previously $[16,17]$. Although these EV types have often been associated with large outbreaks due to appearance of new recombinant forms, no outbreaks occurred over the study period in Scotland.

In addition, six different species $A$ EV serotypes (CAV2, $\mathrm{CAV}_{4}, \mathrm{CAV} 6, \mathrm{CAV}_{10}, \mathrm{CAV} 16$ and $\mathrm{EV}_{71}$ ) were detected in CSF samples obtained from young children with sepsis-like illness, of which CAV6 was the most common type identified from subjects with HFMD. The number of clinical specimens obtained from individuals with vesicular rash (usually vesicular fluid samples obtained from individuals with likely HFMD) was approximately 10 -fold less (approximately 100 samples per year) than the number of CSF samples obtained from individuals with suspected meningitis. Typically, surveillance data from the UK and elsewhere in Europe are restricted to EV-infected individuals admitted to hospital; those presenting with HFMD are often diagnosed by their general practitioner without laboratory testing. Information on the epidemiology of HFMD in Europe is therefore very limited. However, detection of species A EV including $\mathrm{EV}_{71}$ in CSF samples (nine cases) and CAV6 in CSF (one case), vesicular swabs (12 cases) and sewage (14/61) indicates that these viruses are circulating in Scotland, and could lead to outbreaks as previously reported in Finland and France $[18,19]$.

Further evidence for the widespread circulation of EV species $A$ variants is provided by the frequent detection of $\mathrm{CAV}_{2}, \mathrm{CAV}_{4}, \mathrm{CAV}_{5}, \mathrm{CAV} 6$ and $\mathrm{CAV}_{10}$ in waste water collected in Edinburgh. However, as shown previously, some of these viruses may not always cause symptomatic infections leading to hospitalisation [3]. For example, $\mathrm{CAV}_{4}$ and $\mathrm{CAV}_{10}$ were among the EV types frequently detected in faecal samples collected from healthy Norwegian infants [20]. On the other hand, both HFMD and severe $E V_{71}$ infections are spreading in Asia, as exemplified by a recent outbreak in Cambodia associated with $95 \%$ mortality. To identify potential global spread, surveillance in the UK and elsewhere in Europe is essential, including testing of community-collected samples as recently carried out in France as part of large prospective observational study [19]. Detection of $E V$ in sewage could provide the earliest indications of such spread, although the sheer diversity of circulating EV types at any one time prevented identification of a clear temporal match between types identified in clinical and sewage surveillance specimens despite the large number of sequences obtained from the two sample sets.

Despite the adoption of PCR-based methods for virus detection, no species C EV were identified in any of the clinical specimens. However, species C EV serotypes were abundant in sewage in the Edinburgh, including $C A V_{1}, C A V_{13}, C A V_{19}, C A V_{22}$ and a possible new EV type. Species $C$ enteroviruses were also the most commonly detected viruses in sewage screening on the Philippines [21]. This dichotomy is best explained by a specifically lower pathogenicity of non-polio EV-C variants [3]. Furthermore, all circulating vaccine-derived PV (cVDPV) have been shown to be recombinants of oral polio vaccine (OPV) strains and co-circulating species C EV [22,23]. This is the first report of the existence of these less pathogenic species C non-PV EV in sewage in Europe. The authors speculate that they may serve as a reservoir for recombination and thus drive the emergence of recombinant cVDPVs in areas where OPV is still used.

Furthermore, EV68 was the only species D virus identified in clinical specimens. It has recently been associated with cases of severe respiratory tract infections in Europe [24] and elsewhere, and thus this finding was not unexpected. However, EV68 infections are generally under-recognised and underreported because the virus has an HRV-like 5'NCR and will often be reported as HRV infections. No species D EV were identified in our sewage screening despite the use of a sensitive $P C R$ with species D-specific VP1 primers [12]. Due to the potential recent emergence of new species $D$ EV types including EV111 and EV120 from sub-Saharan Africa, EV surveillance targeting EV-D types is also relevant. For example, EV94 within species D was identified in the Democratic Republic of the Congo from an individual with acute flaccid paralysis in 2007 and has since been shown to circulate widely in northern Europe based on seroprevalence studies [25].

\section{Diversity of parechoviruses}

In contrast to the diversity of EV detected in CSF samples, all but two of the $68 \mathrm{HPeV}$-positive CSF samples detected over the eight-year period were $\mathrm{HPeV}_{3}$ (the exceptions being one type 1 and one type 5). $\mathrm{HPeV}_{3}$ was the most frequently identified picornavirus type in CNS-related infection in this study, and exclusively 
seen in young children under the age of three months (Figure 1). The biannual cycle of $\mathrm{HPeV}_{3}$ infections observed in this study (Figure 1) is consistent with previous reports of much higher frequencies of $\mathrm{HPeV}_{3}$ infections occurring in even-numbered years in northern Europe $[1,5,26,27]$. In addition, $\mathrm{HPeV}_{3}$ was absent in the sewage water collected in Edinburgh in 2009 and only appeared in sewage one week before the first clinical case was diagnosed in early 2010 (Figure 4). Although more extensive sewage surveillance data is required, this striking correlation demonstrates the potential value of environmental surveillance in detecting changes in HPeV (and other virus) circulation and in anticipation of its subsequent clinical presentations. This is particularly relevant in the case of $\mathrm{HPeV}_{3}$ as, despite being now well recognised as a major cause of severe neonatal sepsis-like illness occasionally leading to fatality [26], routine screening of children with sepsis-like illness is infrequent in the UK and elsewhere. $\mathrm{HPeV}$ infections are still considerably under-diagnosed.

\section{Acknowledgments}

The authors are grateful to Richard Johnson and Paul Banfield at Veolia Water Outsourcing Ltd, Edinburgh for provision of wastewater filtrate for the study.

\section{Conflict of interest}

None declared.

\section{Authors' contributions}

Heli Harvala and Peter Simmonds conceived the study. Heli Harvala analysed the data and wrote the manuscript. Naomi Gadsby, Pamela Moleneaux and Peter Simmonds provided contribution to the manuscript, and all the authors approved the final version. Joe Calvert, Carol McWilliams Leitch and Peter Simmonds organised and performed the sewage collection. All the authors assisted with enterovirus and parechovirus typing.

\section{References}

1. Harvala H, McLeish N, Kondracka J, McIntyre CL, McWilliamLeitch EC, Templeton K, et al. Comparison of human parechovirus and enterovirus detection frequencies in cerebrospinal fluid samples collected over a 5-year period in Edinburgh - HPeV type 3 identified as the most common picornavirus type. J Med Virol. 2011;839(5):889-96. http:// dx.doi.org/10.1002/jmv.22023

2. Solomon T, Lewthwaite P, Perera D, Cardosa MJ, McMinn P, Ooi MH. Virology, epidemiology, pathogenesis, and control of enterovirus 71. Lancet Infect Dis. 2010:10(11);778-90. http:// dx.doi.org/10.1016/S1473-3099(10)70194-8

3. Melnick JL. Enterovirus: Polioviruses, coxsackieviruses, echoviruses and newer enteroviruses. In: Fields BN, Knipe DM, Howley PM, editors. Fields Virology. Philadelphia: LippincottRaven Publishers; 2007.

4. Knowles NJ, Hovi T, Hyypiä T, King AM, Lindberg AM, Pallansch MA, et al. Picornaviridae. In: King AM, Adams MJ, Carstens EB, Lefkowitz EJ, editors. Virus Taxonomy: Classification and Nomenclature of Viruses. Ninth Report of the International Committee on Taxonomy of Viruses. San Diego: Elsevier; 2012. p.855-80.

5. Harvala H, Wolthers K, Simmonds P. Parechoviruses in children: understanding a new infection. Curr Opin Infect Dis. 2010;23(3):224-30. http://dx.doi.org/10.1097/ QCO.obo13e32833890ca
6. Hyypiä T, Horsnell C, Maaronen M, Khan M, Kalkkinen N, Auvinen $\mathrm{P}$, et al. A distinct picornavirus group identified by sequence analysis. Proc Natl Acad Sci U S A. 1992;89(18):884751. http://dx.doi.org/10.1073/pnas.89.18.8847

7. Oberste M, Schnurr D, Maher K, al Busaidy S, Pallansch M. Molecular identification of new picornaviruses and characterization of a proposed enterovirus 73 serotype. J Gen Virol. 2001;82(Pt 2):409-16.

8. Welch JB, McGowan K, Searle B, Gillon J, Jarvis LM, Simmonds P. Detection of enterovirus viraemia in blood donors. Vox Sang. 2001;80(4):211-5. http://dx.doi. org/10.1046/j.1423-0410.2001.00035.x

9. Dierssen U, Rehren F, Henke-Gendo C, Harste G, Heim A. Rapid routine detection of enterovirus RNA in cerebrospinal fluid by a one-step real-time RT-PCR assay. J Clin Virol. 2008;42(1):58-64. http://dx.doi.org/10.1016/j.jcv.2007.11.016

10. Bennett S, Harvala H, Witteveldt J, McWilliam Leitch EC, McLeish N, Templeton K, et al. Rapid Simultaneous Detection of Enterovirus and Parechovirus RNAs in Clinical Samples by One-Step Real-Time Reverse Transcription-PCR Assay. J Clin Microbiol. 2011;49(7):2620-4. http://dx.doi.org/10.1128/ JCM.02445-10

11. Harvala H, Robertson I, McWilliam Leitch C, Chieochansin T, Templeton K, Simmonds P. Aetiological role of human parechovirus type 3 in neonatal sepsis identified by direct typing assay on cerebrospinal fluid. J Infect Dis. 2009;199(12):1753-60. http://dx.doi.org/10.1086/599094

12. McLeish N, Witteveldt J, Clasper L, McIntyre C, McWilliam Leitch EC, Hardie A, et al. Development and assay of RNA transcripts of enteroviruses species A-D, rhinovirus species A-C and human parechovirus: assessment of assay sensitivity and specificity of real-time screening and typing methods. J Clin Microbiol. 2012;50(9):2910-7. http://dx.doi.org/10.1128/ JCM.01172-12

13. Symonds EM, Griffin DW, Breitbart M. Eukaryotic viruses in wastewater samples from the United States. Appl Environ Microbiol. 2009;75(5):1402-9. http://dx.doi.org/10.1128/ AEM.01899-08

14. Harvala H, McIntyre CL, McLeish NJ, Kondracka J, Palmer J, Molyneaux $P$, et al. High detection frequency and viral loads of human rhinovirus species $A$ to $C$ in fecal samples; diagnostic and clinical implications. J Med Virol. 2012;84(3):536-42. http://dx.doi.org/10.1002/jmv.23203

15. Viskari HR, Koskela P, Lonnrot M, Luonuansuu S, Reunanen A, Baer $M$, et al. Can enterovirus infections explain the increasing incidence of type 1 diabetes? Diabetes Care. 2000;23(3):414-6. http://dx.doi.org/10.2337/diacare.23.3.414

16. Maguire HC, Atkinson P, Sharland M, Bendig J. Enterovirus infections in England and Wales: laboratory surveillance data: 1975 to 1994 . Commun Dis Public Health. 1999;2(2):122-5.

17. Khetsuriani N, Lamonte-Fowlkes A, Oberste S, Pallansch MA. Enterovirus surveillance--United States, 1970-2005. MMWR Surveill Summ. 2006;55(8):1-20.

18. Osterback R, Vuorinen T, Linna M, Susi P, Hyypia T, Waris M. Coxsackievirus A6 and hand, foot, and mouth disease, Finland. Emerg Infect Dis. 2009;15(9):1485-8. http://dx.doi.org/10.3201/ eid1509.090438

19. Mirand A, Henquell C, Archimbaud C, Ughetto S, Antona D, Bailly JL, et al. Outbreak of hand, foot and mouth disease herpangina associated with coxsackievirus $\mathrm{A} 6$ and $\mathrm{A} 10$ infections in 2010, France: a large citywide, prospective observational study. Clin Microbiol Infect. 2012;18(5):E110-8. http://dx.doi.org/10.1111/j.1469-0691.2012.03789.x

20. Witso E, Palacios G, Cinek O, Stene LC, Grinde B, Janowitz $D$, et al. High prevalence of human enterovirus a infections in natural circulation of human enteroviruses. J Clin Microbiol. 2006;44(11):4095-100. http://dx.doi.org/10.1128/ JCM.00653-06

21. Apostol LN, Imagawa T, Suzuki A, Masago Y, Lupisan S, Olveda $R$, et al. Genetic diversity and molecular characterization of enteroviruses from sewage-polluted urban and rural rivers in the Philippines. Virus Genes. 2012;45(2):207-17. http://dx.doi. org/10.1007/s11262-012-0776-z

22. Combelas N, Holmblat B, Joffret ML, Colbere-Garapin F, Delpeyroux F. Recombination between poliovirus and coxsackie A viruses of species C: a model of viral genetic plasticity and emergence. Viruses. 2011;3(8):1460-84. http:// dx.doi.org/10.3390/v3081460

23. Sadeuh-Mba SA, Bessaud M, Massenet D, Joffret ML, Endegue MC, Njouom R, et al. Enteroviruses in Cameroon and neighbouring countries: high frequency and diversity of species C enteroviruses. J Clin Microbiol. 2012;51(3):759-70. http://dx.doi.org/10.1128/JCM.02119-12

24. Meijer A, van der Sanden S, Snijders BE, Jaramillo-Gutierrez $G$, Bont L, van der Ent CK, et al. Emergence and epidemic occurrence of enterovirus 68 respiratory infections in The 
Netherlands in 2010. Virology. 2012;423(1):49-57. http://

dx.doi.org/10.1016/j.virol.2011.11.021

25. Smura TP, Junttila N, Blomqvist S, Norder H, Kaijalainen S, Paananen A, et al. Enterovirus 94, a proposed new serotype in human enterovirus species D. J Gen Virol. 2007;88(3):849-58. http://dx.doi.org/10.1099/vir.0.82510-0

26. Schuffenecker I, Javouhey E, Gillet Y, Kugener B, Billaud $\mathrm{G}$, Floret D, et al. Human parechovirus infections, Lyon, France, 2008-10: evidence for severe cases. J Clin Virol. 2012;54(4):337-41. http://dx.doi.org/10.1016/j.jcv.2012.04.016

27. Wolthers KC, Benschop KS, Schinkel J, Molenkamp R,

Bergevoet RM, Spijkerman IJ, et al. Human parechoviruses as an important viral cause of sepsislike illness and meningitis in young children. Clin Infect Dis. 2008;47(3):358-63. http:// dx.doi.org/10.1086/589752 